\title{
Sagittal Abdominal Diameter as a Screening Tool in Clinical Research: Cutoffs for Cardiometabolic Risk
}

\author{
U. Risérus, ${ }^{1}$ U. de Faire, ${ }^{2}$ L. Berglund, ${ }^{3}$ and M.-L. Hellénius ${ }^{4}$ \\ ${ }^{1}$ Clinical Nutrition and Metabolism, Department of Public Health and Caring Sciences, Uppsala University, 75185 Uppsala, Sweden \\ ${ }^{2}$ Division of Cardiovascular Epidemiology, Institute of Environmental Medicine, Karolinska Institute and Department of Cardiology, \\ Karolinska University Hospital, 17177 Stockholm, Sweden \\ ${ }^{3}$ Uppsala Clinical Research Center (UCR), Uppsala University, 75183 Uppsala, Sweden \\ ${ }^{4}$ Department of Medicine, Karolinska Institute, Karolinska University Hospital, 17176 Stockholm, Sweden
}

Correspondence should be addressed to U. Risérus, ulf.riserus@pubcare.uu.se

Received 19 August 2009; Revised 27 December 2009; Accepted 3 February 2010

Academic Editor: Karen Charlton

Copyright (c) 2010 U. Risérus et al. This is an open access article distributed under the Creative Commons Attribution License, which permits unrestricted use, distribution, and reproduction in any medium, provided the original work is properly cited.

\begin{abstract}
Background. Waist girth and BMI are commonly used as markers of cardiometabolic risk. Accumulating data however suggest that sagittal abdominal diameter (SAD) or "abdominal height" may be a better marker of intra-abdominal adiposity and cardiometabolic risk. We aimed to identify cutoffs for SAD using a cardiometabolic risk score. Design. A population-based crosssectional study. Methods. In 4032 subjects (1936 men and 2096 women) at age 60, different anthropometric variables (SAD, BMI, waist girth, and waist-to-hip ratio) were measured and cardiometabolic risk score calculated. ROC curves were used to assess cutoffs. Results. Among men SAD showed the strongest correlations to the majority of the individual risk factors; whereas in women SAD was equal to that of waist girth. In the whole sample, the area under the ROC curve was highest for SAD. The optimal SAD cutoff for an elevated cardiometabolic risk score in men was $\sim 22 \mathrm{~cm}(95 \% \mathrm{CI} ; 21.6$ to 22.8$)$ and in women $\sim 20 \mathrm{~cm}(95 \% \mathrm{CI}$; 19.4 to 20.8). These cutoffs were similar if the Framingham risk score was used. Conclusions. These cutoffs may be used in research and screening to identify "metabolically obese" men who would benefit from lifestyle and pharmacological interventions. These results need to be verified in younger age groups.
\end{abstract}

\section{Introduction}

Anthropometric measures are widely used as simple markers to identify subjects at risk of cardiovascular disease and diabetes. A high BMI reflects generalized overweight and obesity and is associated with cardiovascular morbidity and mortality. Abdominal obesity, especially visceral obesity, is however more hazardous than generalized obesity [1]. Abdominal fat distribution is commonly measured as waist girth or waist/hip ratio, measures that have been shown to predict cardiovascular disease independent of BMI $[2,3]$. In fact, a recent large meta-analysis suggests that BMI is not a significant predictor of cardiovascular mortality except in patients with severe obesity [4].

More recently, "abdominal height" or sagittal abdominal diameter (SAD) has shown to be strongly associated with glucose intolerance [5], cardiovascular risk [5-9], and mortality [10-13], independently of other anthropometric measures. SAD is also an excellent estimate of visceral fat [14-16] implying that SAD might be a particularly good marker of insulin resistance, which also has been demonstrated in men [17] and women [18]. Despite these promising data, the role of SAD has been overlooked whereas waist girth has received more attention $[5,19-21]$. We have previously reported that SAD was a better predictor of cardiometabolic risk compared to waist girth and other conventional measures [7]. Now we thus need cutoffs for SAD, data that needs to be derived from a large population-based sample comparing SAD with other measures of abdominal obesity. It is necessary to identify cutoffs in order to use SAD either in research as a screening tool or in the clinic to identify individuals with elevated cardiometabolic risk. 
Hence, the aim of this study was to identify optimal cutoffs for SAD to be used in future prospective studies and research. In a population-based sample of Swedish subjects aged 60 we compared SAD with other more conventional anthropometric measures.

\section{Material and Methods}

2.1. Subjects. In 1997 to 1999 , every third man and woman ( $n=5460$ in total, 2681 men and 2779 women) living in Stockholm County, Sweden, born between 1937 and 1938, was invited to participate in a health screening survey. Of all invited, 4228 individuals agreed to participate (2036 men and 2192 women, $78 \%$ response rate), representing the general population of subjects aged 60 [22]. In the current study we excluded subjects without complete data on all anthropometric, metabolic and cardiovascular variables, resulting in 4032 subjects (1936 men, and 2096 women) that comprise the current study population.

2.2. Clinical Investigation. All subjects underwent a physical examination including anthropometry and blood biochemistry after an overnight fast [22]. Body weight was measured using an electronic scale to the nearest $0.1 \mathrm{~kg}$, with the subjects wearing light clothing and no shoes. Height was measured without shoes to nearest $0.5 \mathrm{~cm}$. BMI was calculated as weight $(\mathrm{kg})$ divided by height $(\mathrm{m})$ squared. SAD (anteroposterior) or "abdominal height" was measured after a normal expiration to nearest $0.1 \mathrm{~cm}$ in supine position with straight legs on a firm examination table, without clothes in the measurement area. At the level of iliac crest $\left(\mathbf{L}_{4-5}\right)$.SAD was measured using a ruler and water level. $\mathrm{SAD}$ was the distance between the examination table up to the horizontal level. Intraobserver variation (coefficient of variation) for SAD was $1.6 \%$, and intrasubject variation was $2.7 \%$ [23]. Waist girth was measured according to the WHO in underwear in standing position after normal expiration, midway between the lower rib margin and the iliac crest and hip girth was measured at symphysis trochanter level [24].

Systolic and diastolic blood pressures were measured twice after 5 minutes rest in supine position, using a digital blood pressure monitor (HEM 711, OMROM Health Care) and the mean of the two values was calculated [22].

Venous blood was drawn from antecubital vein after overnight fasting. All blood samples were analyzed online during the study. Cholesterol and triglycerides in serum were analyzed by enzymatic methods (Bayer diagnostics, Tarrytown, USA) [25, 26]. HDL-cholesterol in serum was measured enzymatically after isolation of LDL and VLDL (Boehringer Mannheim GmbH, Germany) and LDLcholesterol was estimated using Friedewald's method [27]. ApoB and apoA-I were determined by an immunoturbidimetric method [28]. Serum glucose was measured with an enzymatic colorimetric test (Bayer Diagnostics, Tarrytown, USA) [29]. Serum insulin levels were determined using the ELISA technique (Boeringer Mannheim GmbH, Diagnostica, Germany). Plasma fibrinogen was measured with a functional spectrophotometric test (Boehringer Mannheim,
Germany) [30]. $\gamma$-glutamyltransferase in serum was determined using an enzymatic colorimetric test (Bayer Diagnostics, Tarrytown, USA).

2.3. Statistical Analyses. Variables that were skewed were logarithmically transformed before statistical analysis. All metabolic and anthropometric variables were normally distributed after transformation. Pearson's correlation coefficients were used to investigate the associations between anthropometric and metabolic variables. An established cardiometabolic risk score (reflecting metabolic aberrations related to the metabolic syndrome) was calculated, and comprised serum triglycerides, HDL-cholesterol, fasting glucose, fasting insulin, and supine systolic and diastolic blood pressures [7]. Each item was converted to quintiles. For all items except HDL-cholesterol the $20 \%$ lowest values were given the quintile value 1 and so on. HDL-cholesterol was given quintile numbers in the reverse order, so that the $20 \%$ with the lowest values were given the quintile value 5 , and so forth. The risk score was calculated as the sum of quintiles over all items. The sum may span from 6 to 30 and a value above the 80th percentile within each sex was defined as an elevated cardiometabolic risk score [7]. In addition to the cardiometabolic risk score, we also identified cutoffs by using the Framingham risk score (http://www.nhlbi.nih.gov/guidelines/cholesterol/risk tbl.htm). We defined an elevated Framingham risk score as the highest quintile per gender, thus we used a similar definition as for the cardiometabolic risk score to facilitate comparison. From the Framingham point scores, total Framingham risk was estimated as a 10-year risk (\%) of having cardiovascular disease.

For each anthropometric measure, a Receiver Operating Characteristic (ROC) curve was calculated for prediction of an elevated cardiometabolic risk score, within each sex. The c-statistic (area under the ROC curve) and an optimal cutoff, calculated from the Youden index [31] (the maximal sum of sensitivity and specificity), are presented. The sensitivity and specificity (with confidence intervals) based on the optimal cutoff are also presented. Confidence intervals for the Cutoffs were assessed with the bootstrap normal approximation method [32]. We used 10000 bootstrap samples. Confidence intervals for the c-statistic and significance tests for comparison of c-statistics from different anthropometric measures were calculated according to the method described by DeLong et al. [33].

$P<.05$ was regarded as statistically significant. JMP and SAS version 9 software packages were used for statistical analyses (SAS Institute Inc., Cary, NC, USA).

\section{Results}

The mean (SD) cardiometabolic risk score was 14 (5) for men and 10 (5) for women.

Baseline characteristics of subjects with data on all variables are presented as quintiles for men and women, respectively (Tables 1 and 2). All anthropometric measures were significantly correlated with all individual risk factors 
TABLE 1: Individual anthropometric measures and cardiometabolic risk factors divided into quintiles in men.

\begin{tabular}{|c|c|c|c|c|c|}
\hline & Quintile 1 & Quintile 2 & Quintile 3 & Quintile 4 & Quintile 5 \\
\hline $\mathrm{SAD}(\mathrm{cm})$ & $18.1 \pm 2.1$ & $18.8 \pm 2.2$ & $19.4 \pm 2.3$ & $20.5 \pm 2.6$ & $22.0 \pm 3.0$ \\
\hline Waist $(\mathrm{cm})$ & $79.0 \pm 8.7$ & $82.5 \pm 9.0$ & $85.0 \pm 10.4$ & $89.8 \pm 10.9$ & $95.7 \pm 12.4$ \\
\hline Waist-to-hip ratio & $0.8 \pm 0.05$ & $0.81 \pm 0.05$ & $0.82 \pm 0.05$ & $0.85 \pm 0.06$ & $0.88 \pm 0.06$ \\
\hline BMI & $24.2 \pm 3.5$ & $25.3 \pm 3.6$ & $26.1 \pm 4.0$ & $7.7 \pm 4.5$ & $29.8 \pm 5.0$ \\
\hline Glucose $(\mathrm{mmol} / \mathrm{L})^{*}$ & $4.7 \pm 0.4$ & $4.9 \pm 0.5$ & $5.2 \pm 0.8$ & $5.5 \pm 1.3$ & $6.2 \pm 2.3$ \\
\hline Insulin $(\mathrm{mU} / \mathrm{L})^{*}$ & $6.1 \pm 4.0$ & $7.5 \pm 3.1$ & $9.4 \pm 5.2$ & $10.6 \pm 5.1$ & $14.5 \pm 7.9$ \\
\hline Triglycerides $(\mathrm{mmol} / \mathrm{L})^{*}$ & $0.8 \pm 0.2$ & $1.0 \pm 0.4$ & $1.1 \pm 0.5$ & $1.4 \pm 0.6$ & $1.9 \pm 0.9$ \\
\hline LDL cholesterol $(\mathrm{mmol} / \mathrm{L})^{*}$ & $3.7 \pm 0.9$ & $3.8 \pm 0.9$ & $3.9 \pm 1.0$ & $4.0 \pm 0.9$ & $4.1 \pm 1.0$ \\
\hline HDL cholesterol $(\mathrm{mmol} / \mathrm{L})^{*}$ & $1.9 \pm 0.3$ & $1.7 \pm 0.4$ & $1.7 \pm 0.4$ & $1.5 \pm 0.4$ & $1.4 \pm 0.3$ \\
\hline ApoB/ApoA1 & $0.56 \pm 0.1$ & $0.61 \pm 0.2$ & $0.66 \pm 0.2$ & $0.71 \pm 0.2$ & $0.81 \pm 0.2$ \\
\hline Systolic BP (mm Hg)* & $114 \pm 11$ & $124 \pm 15$ & $136 \pm 19$ & $142 \pm 20$ & $153 \pm 20$ \\
\hline Diastolic BP $(\mathrm{mm} \mathrm{Hg})^{*}$ & $72 \pm 6$ & $77 \pm 7$ & $82 \pm 9$ & $85 \pm 9$ & $89 \pm 9$ \\
\hline Fibrinogen $(\mathrm{g} / \mathrm{L})$ & $2.7 \pm 0.6$ & $2.9 \pm 0.7$ & $3.1 \pm 0.7$ & $3.2 \pm 0.8$ & $3.4 \pm 0.8$ \\
\hline$\gamma$-Glutamyltransferase $(\mu \mathrm{kat} / \mathrm{L})$ & $0.39 \pm 0.7$ & $0.40 \pm 0.3$ & $0.48 \pm 0.6$ & $0.7 \pm 0.9$ & $0.7 \pm 1.0$ \\
\hline
\end{tabular}

Data are Mean $\pm \mathrm{SD} .{ }^{*}$ Risk factors included in the cardiometabolic risk score.

TABLE 2: Individual anthropometric measures and cardiometabolic risk factors divided into quintiles in women.

\begin{tabular}{|c|c|c|c|c|c|}
\hline & Quintile 1 & Quintile 2 & Quintile 3 & Quintile 4 & Quintile 5 \\
\hline $\mathrm{SAD}(\mathrm{cm})$ & $19.4 \pm 2.0$ & $20.4 \pm 2.0$ & $21.5 \pm 2.3$ & $22.1 \pm 2.4$ & $23.8 \pm 2.7$ \\
\hline Waist $(\mathrm{cm})$ & $90.0 \pm 8.3$ & $94.1 \pm 8.0$ & $97.9 \pm 8.3$ & $100.3 \pm 8.0$ & $106.0 \pm 10.3$ \\
\hline Waist-to-hip ratio & $0.91 \pm 0.05$ & $0.93 \pm 0.05$ & $0.96 \pm 0.05$ & $0.97 \pm 0.05$ & $0.99 \pm 0.05$ \\
\hline BMI & $24.3 \pm 2.6$ & $25.6 \pm 2.9$ & $27.0 \pm 3.2$ & $27.8 \pm 3.4$ & $30.0 \pm 3.8$ \\
\hline Glucose $(\mathrm{mmol} / \mathrm{L})^{*}$ & $5.0 \pm 0.7$ & $5.3 \pm 0.9$ & $5.7 \pm 1.4$ & $6.0 \pm 1.8$ & $6.9 \pm 2.5$ \\
\hline Insulin $(\mathrm{mU} / \mathrm{L})^{*}$ & $6.4 \pm 3.5$ & $7.9 \pm 3.5$ & $10.2 \pm 4.9$ & $12.7 \pm 9.1$ & $18.1 \pm 10.3$ \\
\hline Triglycerides $(\mathrm{mmol} / \mathrm{L})^{*}$ & $0.8 \pm 0.5$ & $1.1 \pm 0.6$ & $1.3 \pm 0.6$ & $1.7 \pm 0.9$ & $2.3 \pm 1.5$ \\
\hline LDL cholesterol $(\mathrm{mmol} / \mathrm{L})^{*}$ & $3.6 \pm 0.8$ & $3.8 \pm 0.9$ & $3.8 \pm 0.9$ & $3.9 \pm 0.8$ & $3.9 \pm 0.9$ \\
\hline HDL cholesterol $(\mathrm{mmol} / \mathrm{L})^{*}$ & $1.5 \pm 0.3$ & $1.4 \pm 0.3$ & $1.3 \pm 0.3$ & $1.2 \pm 0.3$ & $1.1 \pm 0.2$ \\
\hline ApoB/ApoA1 ratio & $0.65 \pm 0.2$ & $0.72 \pm 0.2$ & $0.80 \pm 0.2$ & $0.84 \pm 0.2$ & $0.89 \pm 0.2$ \\
\hline Systolic blood pressure $(\mathrm{mm} \mathrm{Hg})^{*}$ & $124 \pm 13$ & $136 \pm 16$ & $142 \pm 18$ & $150 \pm 18$ & $159 \pm 18$ \\
\hline Diastolic blood pressure $(\mathrm{mm} \mathrm{Hg})^{*}$ & $78 \pm 7$ & $84 \pm 8$ & $88 \pm 9$ & $91 \pm 9$ & $96 \pm 9$ \\
\hline Fibrinogen $(\mathrm{g} / \mathrm{L})$ & $2.8 \pm 0.8$ & $2.9 \pm 0.8$ & $3.0 \pm 0.7$ & $3.0 \pm 0.7$ & $3.2 \pm 0.7$ \\
\hline$\gamma$-Glutamyltransferase $(\mu \mathrm{kat} / \mathrm{L})$ & $0.59 \pm 0.7$ & $0.64 \pm 0.7$ & $0.74 \pm 0.9$ & $0.9 \pm 0.9$ & $1.13 \pm 1.1$ \\
\hline
\end{tabular}

Data are Mean \pm SD. BP: blood pressure. ${ }^{*}$ Risk factors included in the cardiometabolic risk score.

except to LDL cholesterol for waist girth, WHR, and BMI in men (Table 3). SAD was the only significant predictor of LDL cholesterol, although the correlation was weak (Table 3).

Among the men, 20.4\% were at elevated cardiometabolic risk score, and among the women $22.4 \%$ were defined as having elevated cardiometabolic risk. These figures are in accordance with those reported for this population concerning prevalence of the metabolic syndrome [22]. SAD was more strongly correlated to most risk factors as compared with other anthropometric measures in men whereas in women SAD was not consistently better than other anthropometric measures (Table 3). SAD showed the strongest correlation with the cardiometabolic risk score among men; whereas it was not a better correlate than other anthropometric measures in women. The stronger correlation between SAD and the cardiometabolic risk score was statistically significantly different compared with all other anthropometric measures in men (all $P<.5$ ), but was only significantly different from BMI in women (Table 3).

In men, SAD showed the highest area under the ROC curve which was statistically significant compared with the other measures except BMI $(P=.003, P<.0001$, and $P=.09$ for comparison with waist girth, WHR, and BMI, resp.), and in women $\mathrm{SAD}$ showed together with waist girth the highest area under the ROC curve $(P=.9, P=.3$, and $P<.001$ for comparison between SAD and waist girth, WHR, and BMI, resp.) (Table 4). 
TABLE 3: Correlation coefficients between anthropometric and cardiometabolic variables.

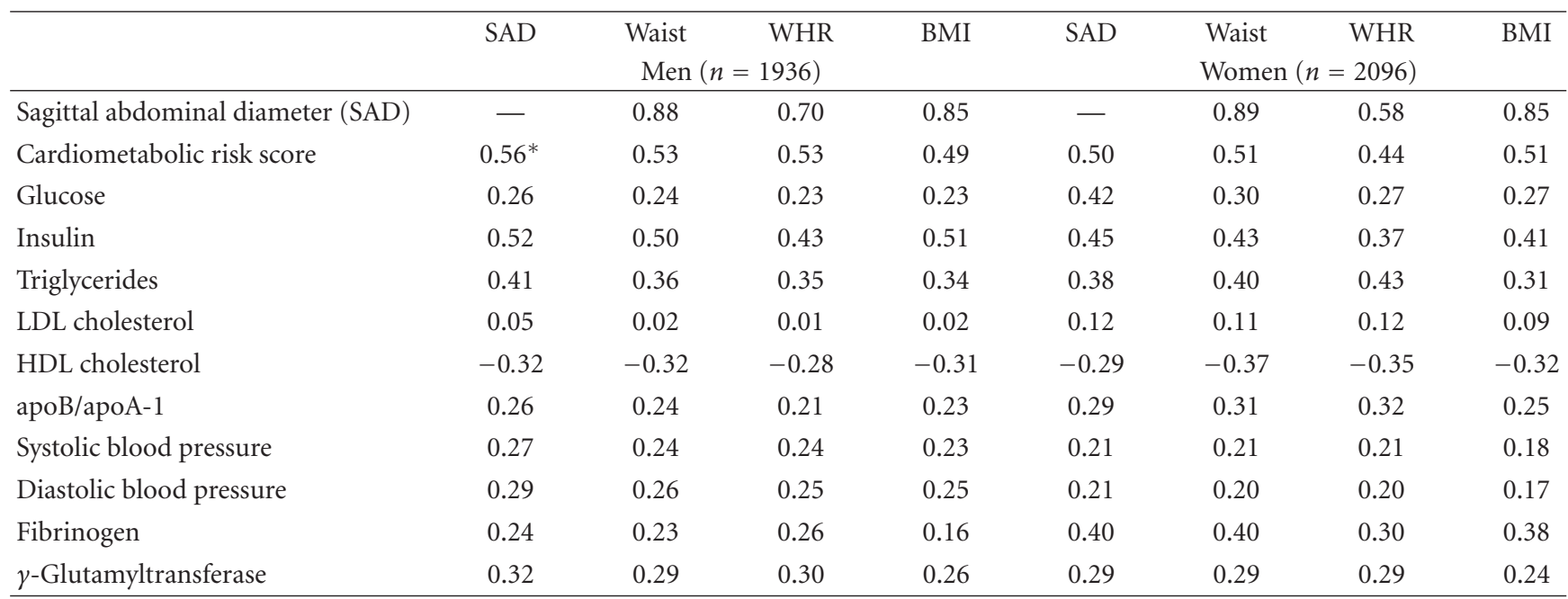

Data are Pearson's correlation coefficients. WHR: waist-to-hip ratio. All correlations were significant $(P<.05)$ except that for waist girth, BMI, and WHR with LDL cholesterol in men. *The correlation between SAD and the cardiometabolic risk score was statistically significantly different compared with all other anthropometric measures in men (all $P<.05$ ), but was only significantly different from BMI in women.

TABLE 4: Area under the ROC curve for different anthropometric measures for identifying individuals at elevated cardiometabolic risk.

\begin{tabular}{llr}
\hline & $\begin{array}{l}\text { Men }(n=1936) \\
\text { AUC under ROC } \\
(95 \% \mathrm{CI})\end{array}$ & $\begin{array}{l}\text { Women }(n=2096) \\
\text { AUC } \\
\text { under ROC } \\
(95 \% \mathrm{CI})\end{array}$ \\
\hline SAD $(\mathrm{cm})$ & $0.80(0.77 ; 0.82)$ & $0.77(0.75 ; 0.80)$ \\
Waist girth $(\mathrm{cm})$ & $0.78(0.75 ; 0.80)$ & $0.77(0.75 ; 0.80)$ \\
Waist-to-hip ratio & $0.74(0.71 ; 0.77)$ & $0.76(0.74 ; 0.79)$ \\
BMI $\left(\mathrm{kg} / \mathrm{m}^{2}\right)$ & $0.78(0.76 ; 0.81)$ & $0.74(0.72 ; 0.77)$ \\
\hline
\end{tabular}

SAD: Sagittal abdominal diameter, CI: confidence interval, BMI: body mass index.

The optimal (95\% CI) SAD cutoff for an elevated calculated cardiometabolic risk score for men was 22.2 (21.6 to 22.8) and for women 20.1 (19.4 to 20.8) (Table 5). The optimal cutoffs for waist girth, WHR, and BMI are shown in Table 5.

To further validate these results, we also assessed optimal cutoffs for SAD using the more established Framingham risk score. The present SAD cutoff of $22 \mathrm{~cm}$ for men was unchanged if replacing the cardiometabolic risk score with Framingham risk score. In women the cutoff was $21 \mathrm{~cm}$ if using the Framingham risk score instead of the cardiometabolic risk score.

The highest quintile of Framingham risk score started at 14 risk points for men, and at 17 risk points for women which corresponded to a $2 \%$ and $5 \% 10$-year risk of cardiovascular disease, respectively. In this population, a SAD of $22 \mathrm{~cm}$ corresponded to $20 \%$ risk in men, and a SAD of $21 \mathrm{~cm}$ corresponded to a $14 \%$ risk in women.

\section{Discussion}

The optimal cutoffs for identifying subjects with an elevated cardiometabolic risk score were $\sim 22 \mathrm{~cm}$ in men and $\sim 20 \mathrm{~cm}$ in women. These figures corresponded to a BMI $\sim 28$ in both sexes. This is the first study using ROC curves to identify cutoffs for SAD in a large population-based sample. Based on a Canadian population in 81 men and 70 women, a SAD > $25 \mathrm{~cm}$ was suggested to be associated with multiple metabolic disorders [5]. Since the sample size of that study was small it may be unwise to compare that study with ours where the cutoffs where lower. Notably, the present SAD cutoff of $22 \mathrm{~cm}$ for men was unchanged if replacing the cardiometabolic risk score with the Framingham risk score, a finding that strengthen the validity of this cutoff. In women the cutoff was however $1 \mathrm{~cm}$ higher if using the Framingham risk score instead of the cardiometabolic risk score.

In men, SAD was significantly more closely correlated with the cardiometabolic risk score versus all other anthropometric measures and also showed slightly stronger correlations with most individual risk factors. In women, SAD was an equally good predictor for an elevated cardiometabolic risk score as waist girth. If one should use only one anthropometric measure, SAD thus may be a good choice to capture elevated cardiometabolic risk. The stronger capacity of SAD to predict cardiometabolic risk in men may be explained by the higher visceral fat content in men 
TABLE 5: Cutoffs for different anthropometric measures in determining an elevated cardiometabolic risk score in men and women.

\begin{tabular}{lll}
\hline & Men $(n=1936)$ & $\begin{array}{l}\text { Women }(n=2096) \\
\text { Mean }(95 \% \mathrm{CI})\end{array}$ \\
\hline Sagittal abdominal diameter $(\mathrm{cm})$ & Mean $(95 \% \mathrm{CI})$ & $20.1(19.4$ to 20.8$)$ \\
Waist girth $(\mathrm{cm})$ & $22.2(21.6$ to 22.8$)$ & $88.4(86.7$ to 90$)$ \\
Waist-to-hip ratio & $100(96.9$ to 101$)$ & $0.82(0.81$ to 0.84$)$ \\
BMI $\left(\mathrm{kg} / \mathrm{m}^{2}\right)$ & $0.97(0.95$ to 0.99$)$ & $27.6(27$ to 28.3$)$ \\
\hline
\end{tabular}

CI: confidence interval, BMI: body mass index.

versus women at a given BMI. This is relevant since visceral adiposity may confer a higher cardiometabolic risk than other fat depots, although this has been debated [34]. Indeed, SAD seems particularly good in capturing visceral fat (which during the supine measurement does not "float out" sideways, as would more be the case for subcutaneous fat) [15, 35]. Also, the cardiometabolic risk score was lower in women (10 versus 14) providing a higher number of men at risk, which could contribute to gender differences observed in this study.

In line with previous data on men, but also to some extent in women, SAD was more closely related to hyperlipidemia [7] and cardiovascular risk [7-9, 36, 37] than BMI, waist girth, and WHR. Recent data also showed that SAD was the best correlate to hypertension $[7,36]$ and plasminogen activating inhibitor-1 [7]. Furthermore, in the Swedish Obese Subjects study the change in SAD was most closely related to the change of metabolic disorders [38]. We have also shown that SAD was a significantly better predictor of insulin resistance than waist girth in men, even independently of BMI and waist girth [17]. Thus, SAD may carry unique information beyond that of other anthropometric measures. Similarly, SAD was the strongest predictor of insulin resistance and CRP levels in healthy women [18]. A recent study in elderly 389 men and 437 women (aged 56-83 years) did however not find that SAD was superior to waist with regard associations with components of the metabolic syndrome [39]. The latter inconsistency might be due to older age groups studied, ethnic, sex, phenotype, or methodological differences.

One possible explanation for a somewhat better predictive capacity of SAD is the higher measurement reliability of SAD compared with waist girth $[23,40]$. SAD may also be the only measure with high reliability in both lean and obese subjects [23]. Again, SAD may more closely reflect visceral adiposity $[14-16,41]$ and thereby better capture cardiometabolic risk [1]. However, it should be noted that SAD also is a valid measure of total abdominal fat [16].

It has been shown that a large waist is a useful tool to detect metabolic disorders $[5,19,20,42]$. These studies did however not compare the predictive capacity with SAD. In one study SAD and waist girth were equally good markers of various metabolic disorders [5]. The present results in a large population do suggest that in men SAD is more strongly associated with individual cardiometabolic risk factors. With regard to mortality, available studies indicate that SAD is a strong predictor of mortality independently of BMI [10-13], especially in younger adults $[11,13]$. However, these studies did not include waist girth or the waist-hip ratio, and only one study included women which showed that SAD was a stronger predictor of mortality than BMI also in women [13]. That study also showed that SAD was good predictor in several ethnic groups.

It has sometimes been argued that waist girth is more practical to measure than SAD. The difference is however negligible. Our results support the use of SAD as a valuable screening tool in research since it is a simple, reliable, and cheap marker to identify men at elevated cardiometabolic risk.

There are limitations of this study. This is a crosssectional study and further prospective analyses will be needed to verify SAD as a predictor of mortality. In addition, the cardiometabolic risk score used have not been established as a determinant of cardiovascular events and mortality. It should however be noted that cutoffs for waist circumference used in current clinical guidelines worldwide are based on cross-sectional data as well [19]. Another relevant limitation is the arbitrary definition of an elevated cardiometabolic risk score, but similar results were obtained if the Framingham risk score was used thus supporting the data. Strengths of the study include the population-based, representative sample of men and women of the same age (age 60). For the latter reason confounding influences of age are avoided. On the other hand, the generalizability to younger age groups decreases. Caucasians comprised the majority of the population but several ethnicities were represented in this study; that is, $19 \%$ was of non-Swedish origin [43]. Notably, SAD seems to be useful in various ethnic groups $[13,44]$ and in a US population, SAD (measured in standing) predicted coronary heart disease across racial groups [13].

\section{Conclusion}

The optimal cutoffs for SAD were $>22 \mathrm{~cm}$ in men and $>20 \mathrm{~cm}$ in women. In line with the previous studies, SAD was the best marker of an elevated cardiometabolic risk score in men, and at least as good as other anthropometric measures in women. Thus, SAD may be a good alternative to waist girth as a simple screening tool in research aiming at recruiting subjects at elevated cardiometabolic risk, especially men. Further evaluation of SAD is warranted in younger age groups. 


\section{Acknowledgments}

This study was supported by grants from the Stockholm County Council, the Swedish Heart and Lung Foundation, and the Swedish Research Council (longitudinal research). The first author was funded by the Swedish Society for Medical Research (SSMF) and NordForsk (Nordic Centre of Excellence in Food, Nutrition and Health, SYSDIET).

\section{References}

[1] P. Björntorp, "Visceral obesity: a "civilization syndrome"” Obesity Research, vol. 1, no. 3, pp. 206-222, 1993.

[2] B. Larsson, K. Svardsudd, L. Welin, L. Wilhelmsen, P. Björntorp, and G. Tibblin, "Abdominal adipose tissue distribution, obesity, and risk of cardiovascular disease and death: 13 year follow up of participants in the study of men born in 1913," British Medical Journal, vol. 288, no. 6428, pp. 14011404, 1984.

[3] T. S. Han, M. E. J. Lean, and J. C. Seidell, "Waist circumference remains useful predictor of coronary heart disease," British Medical Journal, vol. 312, no. 7040, pp. 1227-1228, 1996.

[4] A. Romero-Corral, V. M. Montori, V. K. Somers, et al., "Association of bodyweight with total mortality and with cardiovascular events in coronary artery disease: a systematic review of cohort studies," Lancet, vol. 368, no. 9536, pp. 666678, 2006.

[5] M.-C. Pouliot, J.-P. Despres, S. Lemieux, et al., "Waist circumference and abdominal sagittal diameter: best simple anthropometric indexes of abdominal visceral adipose tissue accumulation and related cardiovascular risk in men and women," American Journal of Cardiology, vol. 73, no. 7, pp. 460-468, 1994.

[6] B. Richelsen and S. B. Pedersen, "Associations between different anthropometric measurements of fatness and metabolic risk parameters in non-obese, healthy, middle-aged men," International Journal of Obesity, vol. 19, no. 3, pp. 169-174, 1995.

[7] M. Öhrvall, L. Berglund, and B. Vessby, "Sagittal abdominal diameter compared with other anthropometric measurements in relation to cardiovascular risk," International Journal of Obesity, vol. 24, no. 4, pp. 497-501, 2000.

[8] H. S. Kahn, H. Austin, D. F. Williamson, and D. Arensberg, "Simple anthropometric indices associated with ischemic heart disease," Journal of Clinical Epidemiology, vol. 49, no. 9, pp. 1017-1024, 1996.

[9] J. Gustat, A. Elkasabany, S. Srinivasan, and G. S. Berenson, "Relation of abdominal height to cardiovascular risk factors in young adults: the Bogalusa heart study," American Journal of Epidemiology, vol. 151, no. 9, pp. 885-891, 2000.

[10] H. S. Kahn, E. J. Simoes, M. Koponen, and R. Hanzlick, "The abdominal diameter index and sudden coronary death in men," American Journal of Cardiology, vol. 78, no. 8, pp. 961964, 1996

[11] J. C. Seidell, R. Andres, J. D. Sorkin, and D. C. Muller, "The sagittal waist diameter and mortality in men: the Baltimore longitudinal study on aging," International Journal of Obesity, vol. 18, no. 1, pp. 61-67, 1994.

[12] J. P. Empana, P. Ducimetiere, M. A. Charles, and X. Jouven, "Sagittal abdominal diameter and risk of sudden death in asymptomatic middle-aged men: the Paris Prospective Study I," Circulation, vol. 110, no. 18, pp. 2781-2785, 2004.
[13] C. Iribarren, J. A. Darbinian, J. C. Lo, B. H. Fireman, and A. S. Go, "Value of the sagittal abdominal diameter in coronary heart disease risk assessment: cohort study in a large, multiethnic population," American Journal of Epidemiology, vol. 164, no. 12, pp. 1150-1159, 2006.

[14] H. Kvist, B. Chowdhury, U. Grangård, U. Tylen, and L. Sjostrom, "Total and visceral adipose-tissue volumes derived from measurements with computed tomography in adult men and women: predictive equations," American Journal of Clinical Nutrition, vol. 48, no. 6, pp. 1351-1361, 1988.

[15] K. van der Kooy, R. Leenen, J. C. Seidell, P. Deurenberg, and M. Visser, "Abdominal diameters as indicators of visceral fat: comparison between magnetic resonance imaging and anthropometry," British Journal of Nutrition, vol. 70, no. 1, pp. 47-58, 1993.

[16] J. L. Clasey, C. Bouchard, C. D. Teates, et al., "The use of anthropometric and dual-energy X-ray absorptiometry (DXA) measures to estimate total abdominal and abdominal visceral fat in men and women," Obesity Research, vol. 7, no. 3, pp. 256-264, 1999.

[17] U. Risérus, J. Ärnlöv, K. Brismar, B. Zethelius, L. Berglund, and B. Vessby, "Sagittal abdominal diameter is a strong anthropometric marker of insulin resistance and hyperproinsulinemia in obese men," Diabetes Care, vol. 27, no. 8, pp. 2041-2046, 2004.

[18] G. Mazzali, V. Di Francesco, E. Zoico, et al., "Interrelations between fat distribution, muscle lipid content, adipocytokines, and insulin resistance: effect of moderate weight loss in older women," American Journal of Clinical Nutrition, vol. 84, no. 5, pp. 1193-1199, 2006.

[19] T. S. Han, E. M. van Leer, J. C. Seidell, and M. E. J. Lean, "Waist circumference action levels in the identification of cardiovascular risk factors: prevalence study in a random sample," British Medical Journal, vol. 311, no. 7017, pp. 14011405, 1995.

[20] J.-P. Després, I. Lemieux, and D. Prud'homme, “Treatment of obesity: need to focus on high risk abdominally obese patients," British Medical Journal, vol. 322, no. 7288, pp. 716720, 2001.

[21] H. Wahrenberg, K. Hertel, B.-M. Leijonhufvud, L.-G. Persson, E. Toft, and P. Arner, "Use of waist circumference to predict insulin resistance: retrospective study," British Medical Journal, vol. 330, no. 7504, pp. 1363-1364, 2005.

[22] M. Halldin, M. Rosell, U. de Faire, and M.-L. Hellénius, "The metabolic syndrome: prevalence and association to leisure-time and work-related physical activity in 60-year-old men and women," Nutrition, Metabolism and Cardiovascular Diseases, vol. 17, no. 5, pp. 349-357, 2007.

[23] K. Nordhamn, E. Södergren, E. Olsson, B. Karlström, B. Vessby, and L. Berglund, "Reliability of anthropometrie measurements in overweight and lean subjects: consequences for correlations between anthropometric and other variables," International Journal of Obesity, vol. 24, no. 5, pp. 652-657, 2000.

[24] World Health Organization, "Expert committee on physical status: the use and interpretation of anthropometry-report of WHO Expert Committee," WHO Tech. Rep. Ser 854, 1995.

[25] C. C. Allain, L. S. Poon, C. S. G. Chan, W. Richmond, and P. C. Fu, "Enzymatic determination of total serum cholesterol," Clinical Chemistry, vol. 20, no. 4, pp. 470-475, 1974.

[26] P. Fossati and L. Prencipe, "Serum triglycerides determined colorimetrically with an enzyme that produces hydrogen peroxide," Clinical Chemistry, vol. 28, no. 10, pp. 2077-2080, 1982. 
[27] W. T. Friedewald, R. I. Levy, and D. S. Fredrickson, "Estimation of the concentration of low-density lipoprotein cholesterol in plasma, without use of the preparative ultracentrifuge," Clinical Chemistry, vol. 18, no. 6, pp. 499-502, 1972.

[28] I. Jungner, S. M. Marcovina, G. Walldius, I. Holme, W. Kolar, and E. Steiner, "Apolipoprotein B and A-I values in 147 576 Swedish males and females, standardized according to the World Health Organization-International Federation of Clinical Chemistry First International Reference Materials," Clinical Chemistry, vol. 44, no. 8, pp. 1641-1649, 1998.

[29] P. Trinder, "Determination of blood glucose using an oxidaseperoxidase system with a non-carcinogenic chromogen," Journal of Clinical Pathology, vol. 22, no. 2, pp. 158-161, 1969.

[30] U. Becker, K. Bartl, and A. W. Wahlefeld, "A functional photometric assay for plasma fibrinogen," Thrombosis Research, vol. 35, no. 5, pp. 475-484, 1984.

[31] W. Youden, "Index for rating diagnostic tests," Cancer, vol. 3, pp. 32-35, 1950.

[32] B. Efron and R. Tibshirani, "An introduction to the bootstrap," in Monographs on Statistics and Applied Probability, D. Cox, D. Hinkley, N. Reid, et al., Eds., pp. 168-177, Chapman \& Hall, New York, NY, USA, 1993.

[33] E. R. DeLong, D. M. DeLong, and D. L. Clarke-Pearson, "Comparing the areas under two or more correlated receiver operating characteristic curves: a nonparametric approach," Biometrics, vol. 44, no. 3, pp. 837-845, 1988.

[34] K. N. Frayn, "Visceral fat and insulin resistance-causative or correlative?" British Journal of Nutrition, vol. 83, supplement 1, pp. S71-S77, 2000.

[35] L. Sjöstrom, L. Lönn, and B. Chowdhury, "The sagittal diameter is a valid marker of the visceral adipose tissue volume," in Progress in Obesity Research: 7, A. Angel, H. Andersson, C. Bouchard, L. Lau, L. Leiter, and R. Mendelson, Eds., pp. 309-319, John Libbey, London, UK, 1996.

[36] P. Strazzullo, G. Barba, F. P. Cappuccio, et al., "Altered renal sodium handling in men with abdominal adiposity: a link to hypertension," Journal of Hypertension, vol. 19, no. 12, pp. 2157-2164, 2001.

[37] L. Kumlin, L. Dimberg, and P. Mårin, "Ratio of abdominal sagittal diameter to height is strong indicator of coronary risk," British Medical Journal, vol. 314, p. 830, 1997.

[38] C. D. Sjöström, L. Lissner, and L. Sjöström, "Relationships between changes in body composition and changes in cardiovascular risk factors: the SOS Intervention Study," Obesity Research, vol. 5, no. 6, pp. 519-530, 1997.

[39] J. Mukuddem-Petersen, M. B. Snijder, R. M. van Dam, et al., "Sagittal abdominal diameter: no advantage compared with other anthropometric measures as a correlate of components of the metabolic syndrome in elderly from the Hoorn Study," American Journal of Clinical Nutrition, vol. 84, no. 5, pp. 9951002, 2006.

[40] H. S. Kahn and D. F. Williamson, "Sagittal abdominal diameter," International Journal of Obesity, vol. 17, no. 11, p. 669, 1993.

[41] K. Asayama, K. Dobashi, H. Hayashibe, et al., "Threshold values of visceral fat measures and their anthropometric alternatives for metabolic derangement in Japanese obese boys," International Journal of Obesity, vol. 26, no. 2, pp. 208 213, 2002.

[42] W. M. Kohrt, J. P. Kirwan, M. A. Staten, R. E. Bourey, D. S. King, and J. O. Holloszy, "Insulin resistance in aging is related to abdominal obesity," Diabetes, vol. 42, no. 2, pp. 273-281, 1993.
[43] P.-E. Wändell, A. Wajngot, U. de Faire, and M.-L. Hellénius, "Increased prevalence of diabetes among immigrants from non-European countries in 60-year-old men and women in Sweden," Diabetes and Metabolism, vol. 33, no. 1, pp. 30-36, 2007.

[44] H. Petersson, A. Daryani, and U. Risérus, "Sagittal abdominal diameter as a marker of inflammation and insulin resistance among immigrant women from the Middle East and native Swedish women: a cross-sectional study," Cardiovascular Diabetology, vol. 6, article 10, 2007. 


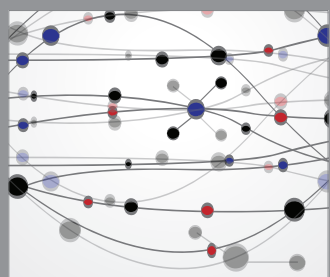

The Scientific World Journal
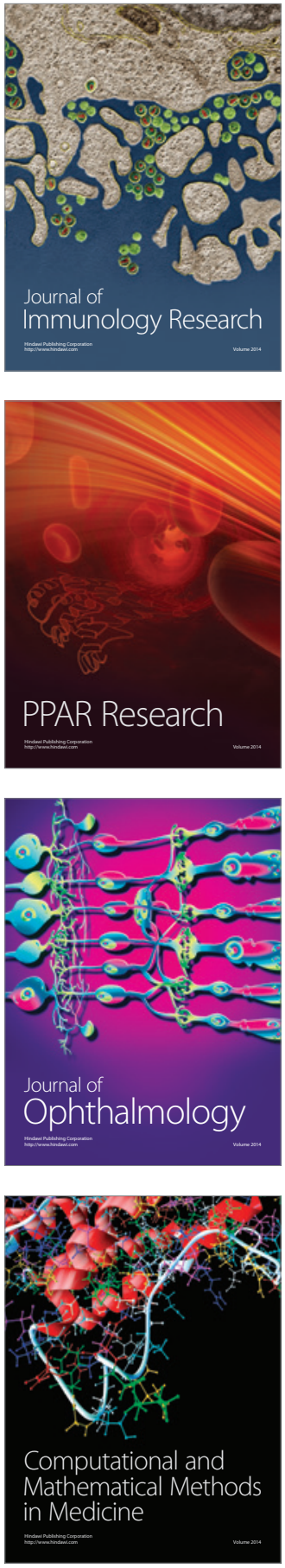

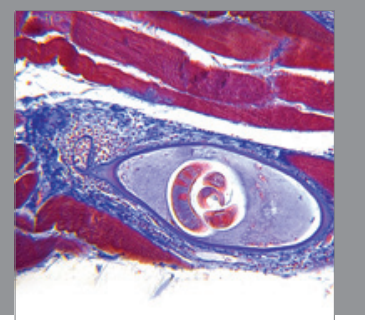

Gastroenterology

Research and Practice
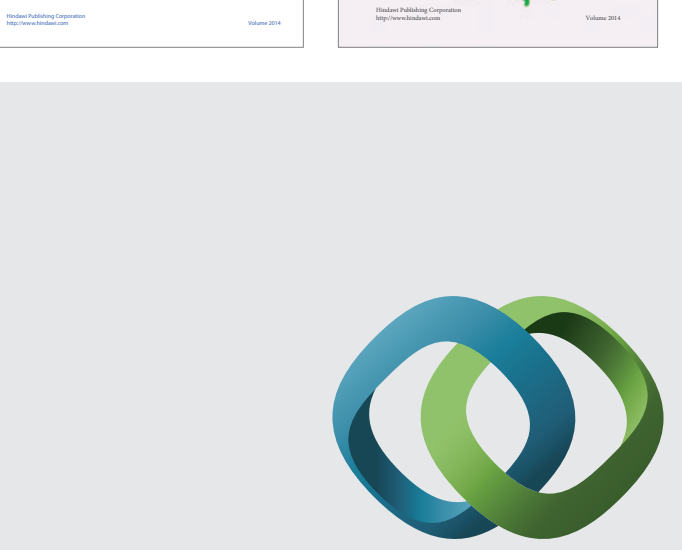

\section{Hindawi}

Submit your manuscripts at

http://www.hindawi.com
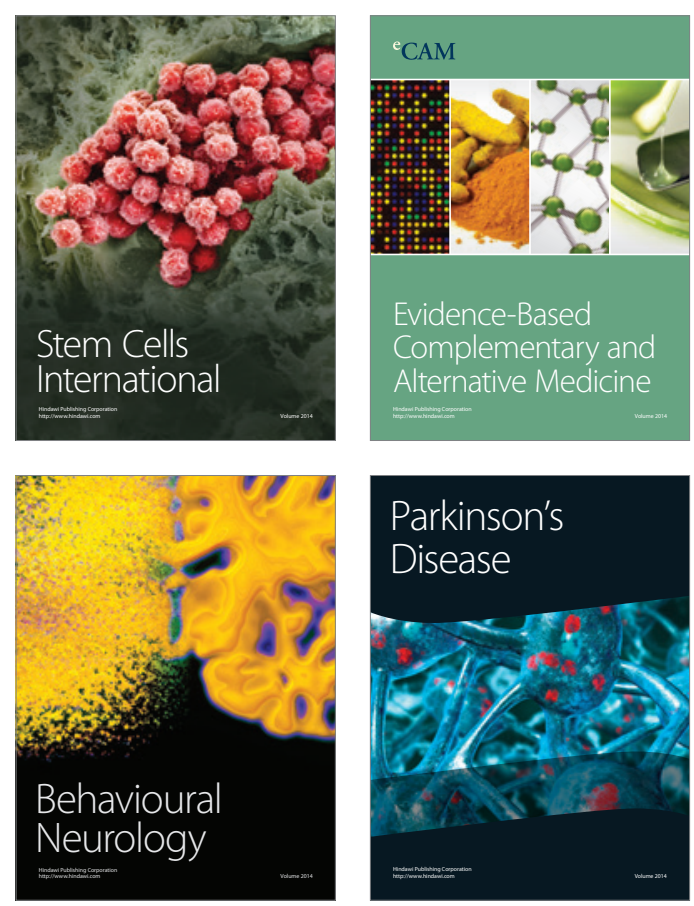

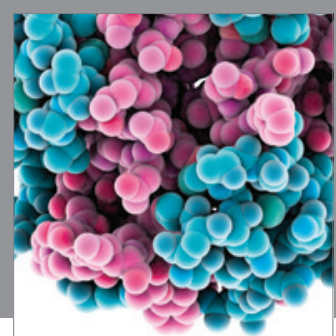

Journal of
Diabetes Research

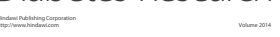

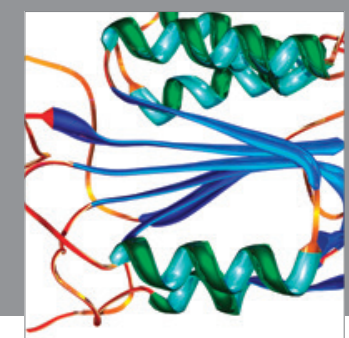

Disease Markers
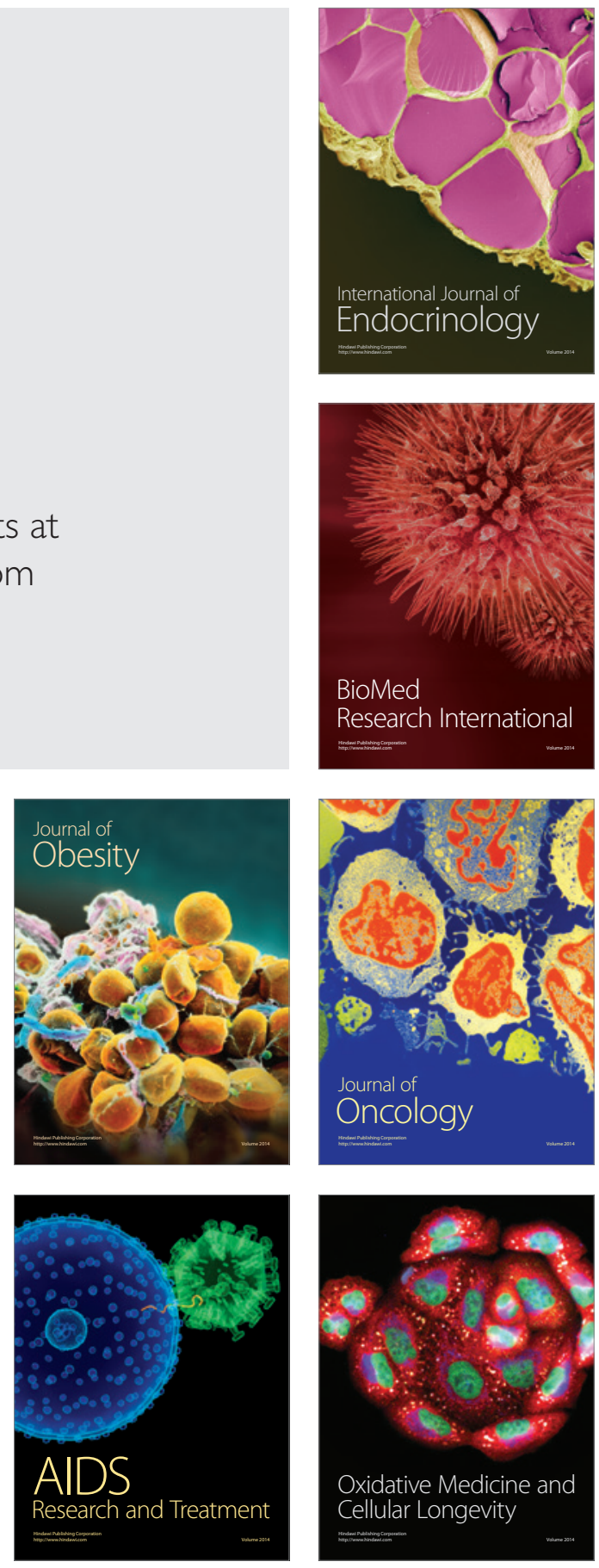